\title{
La aplicabilidad de la normativa jurídica ecuatoriana, en torno a los PDOT cantonales. Estudio de caso del GAD Municipal del cantón Cuenca.
}

\author{
The applicability of Ecuadorian legal regulations, around cantonal \\ PDOTs. Case study of the Municipal GAD of the canton Cuenca.
}

\author{
Juan Andrés Carpio Arévalo ${ }^{1}$
}

Recibido: 10-12-2020 / Revisado: 02-01-2021 /Aceptado: 10-01-2021/ Publicado: 05-02-2021

\begin{abstract}
:
DOI: https://doi.org/10.33262/concienciadigital.v4i1.1.1544

In Ecuador, twelve years after the reform of the Constitution of the Republic and, after several processes of legal reforms and the implementation of new technical and legal instruments at the national level, the cantonal Autonomous Decentralized Governments are in the stage of updating the Plans of Territorial Development and Planning. This article addresses the most relevant aspects in terms of current regulations to structure and develop land use planning plans at the cantonal level, following as a sample type, the case study of the Autonomous Decentralized Government of the Canton Cuenca. To support this research, a qualitative analysis has been carried out, with the review of the main normative bodies of Ecuadorian legislation, as well as different theoretical elements and professional and academic criteria. Likewise, the conducting of interviews with experts in the area, allowed to characterize the process of updating the development plan, land use, land use and management, within the case study. The analysis reveals, in the opinion of the authors, that the Ecuadorian legal framework presents flaws that limit and hinder the exercise of powers and autonomy, guaranteed in the Constitution and the Law, on the use and management of land by the cantonal Autonomous Decentralized Governments.
\end{abstract}

Key words: Normative, Planning, Territorial Development.

\section{Resumen}

En el Ecuador, doce años después de la vigencia de la nueva Constitución de la República $\mathrm{y}$, tras varios procesos de reformas legales y la implementación de nuevos instrumentos técnicos y jurídicos a nivel nacional; los Gobiernos Autónomos Descentralizados cantonales se encuentran en etapa de actualización de los Planes de Desarrollo y Ordenamiento Territorial. Este artículo aborda los aspectos más relevantes en cuanto a la normativa vigente para estructurar y elaborar los planes de ordenamiento territorial a nivel cantonal, siguiendo como muestreo de tipo, el caso de estudio del Gobierno Autónomo

\footnotetext{
${ }^{1}$ Universidad Católica de Cuenca, Posgrado, Maestría en Desarrollo Local mención en Ordenamiento Territorial. Cuenca, Ecuador. juan_andrescarpio@hotmail.com https://orcid.org/0000-0002-5433-9961.
} 
Descentralizado del Cantón Cuenca. Para sustento de la presente investigación, se ha realizado un análisis cualitativo, con la revisión de los principales cuerpos normativos de la legislación ecuatoriana, así como diferentes elementos teóricos y criterios profesionales y académicos. Asimismo, el levantamiento de entrevistas a expertos en el área, permitió caracterizar el proceso de actualización del plan de desarrollo, ordenamiento territorial, uso y gestión de suelo, dentro del caso de estudio. Del análisis se desprende, a criterio de los autores que, el marco jurídico ecuatoriano, presenta falencias que limitan y dificultan el ejercicio de las competencias y autonomía, garantizadas en la Constitución y la Ley, en cuanto al uso y gestión de suelo de los Gobiernos Autónomos Descentralizados cantonales.

Palabras clave: Normativa, Planificación, Desarrollo Territorial.

\section{Introducción:}

El Ecuador durante su vida republicana, ha tenido metamorfosis "kafkianas" en cuanto al ámbito político y gubernamental, donde sin duda los partidos políticos han ejercido un control total en el territorio nacional, llevando la premisa en el quehacer político, caracterizado por ser un conjunto de redes clientelares, donde la coyuntura dividía en parcelas, la organización del Estado. Luego de las reformas constitucionales de los años 1994 y 1997, este monopolio se vio seriamente afectado, tanto más que implicaba una base o inicio para disminuir la afiliación ideológica de los votantes.

En los últimos veinte años el trasfondo político medianamente ha cambiado; pero sin duda con la entrada en vigencia de la Constitución de Montecristi en el año 2008, empiezan a tomar cuerpo figuras políticas hasta el momento desconocidas en el Ecuador, como una real participación ciudadana -al menos teóricamente- y el ejercicio de una democracia directa. A raíz de ello, el garantismo en esta nobel etapa constitucional de la República, otorgó a las regiones, provincias, cantones, etc., la posibilidad de organizarse políticamente a través de Gobiernos Autónomos Descentralizados - GAD, con competencias exclusivas, en virtud del artículo 238 y siguientes de la Carta Magna, así como el artículo 264 ibídem (Constitución de la República del Ecuador, 2008).

En esta línea, la legislación ecuatoriana, faculta a los Gobiernos Autónomos Descentralizados cantonales, crear Planes de Desarrollo y Ordenamiento Territorial (PDOT), donde para su estructuración técnico-jurídica, debe tomar preceptos contenidos en el Código Orgánico de Organización Territorial, Autonomía y Descentralización; el Código Orgánico de Planificación y Finanzas Públicas; la Ley Orgánica de Ordenamiento Territorial, Uso y Gestión de Suelo con su respectivo Reglamento; entre otros cuerpos legales. 
Estudios previos, sugieren una superposición de preceptos jurídicos que, dificultan la correcta aplicabilidad de una planificación de desarrollo y ordenamiento territorial, generando confusión e incertidumbre al momento de elaborar y ejecutar los respectivos instrumentos de planificación (Pauta, F., 2019).

Asimismo, otros análisis demuestran un mediano cumplimiento de las disposiciones legales en cuanto a la construcción e implementación de los Planes de Desarrollo y Ordenamiento Territorial cantonales y, un nulo control de su ejecución (González, M. y Chuquiguanga, C., 2018).

Se han revisado los cuerpos normativos relevantes para la elaboración de políticas públicas y planificación territorial en el ámbito nacional. Esto como fuente primaria. La Constitución y la Ley como fuentes de derecho para la elaboración de los Planes de Desarrollo y Ordenamiento Territorial (PDOT) cantonales. Con ello, se ha logrado tipificar los cuerpos legales que viabilizan la elaboración y ejecución de los PDOT dentro de las competencias de los GAD cantonales.

Para estructurarse una concepción jurídica, es necesario su discusión filosófica. Como dos temas básicos en cuanto al tratamiento del derecho, han resultado las teorías iusnaturalistas y las iuspositivistas. Su eje central de discusión, lo que ha controvertido a los tratadistas, radica en la relación implícita entre el derecho y la moral. Dentro de ésta última acepción (iuspositivismo), surge el concepto del "formalismo jurídico", asociado a la construcción y promulgación misma de los preceptos legales; un conjunto de normas que configuran el orden jurídico de un estado. En la misma línea positivista, se concibe al derecho como un sistema absoluto y completo, el cual no da lugar a los vacíos legales, lo cual por supuesto es ampliamente discutido (Nino, C., 2003, p. 36).

Ante la amplitud y diversidad del territorio ecuatoriano y los sectores productivos, sumado a la extensa normativa de nuestro ordenamiento jurídico, es necesario realizar un análisis contextual sobre la aplicabilidad de los instrumentos jurídicos y su ejecución de orden pragmático en los Planes de Desarrollo y Ordenamiento Territorial (PDOT) cantonales; tomando como caso de estudio al Gobierno Autónomo Descentralizado Municipal del cantón Cuenca.

Es conocido el aforismo jurídico romano que dice: Omnis definitio in jure periculosa est (para indicar que toda definición sobre derecho es subjetiva y riesgosa, inclusive aquellas que se atribuyen a los tratadistas jurídicos); más bien, es acertado lo que indica el autor Manuel Atienza, sobre la ubicuidad del derecho: "El Derecho es un fenómeno omnipresente en nuestras sociedades. Prácticamente no hay ninguna relación social que no esté, o pueda llegar a estar, regulada jurídicamente" (2009, p.15).

Si bien es cierto, el campo de planificación territorial en el país es relativamente nueva, se necesita de diversos factores de cambio estructural, mayor compromiso institucional y 
legislativo, a fin de procurar una simbiosis en el fondo de los instrumentos de planificación. El trabajo conjunto entre los actores sociales, sin duda aporta al desarrollo y el crecimiento de la construcción social. Es necesario mayor involucramiento por parte de los entes gestores de la planificación, ciudadanía y academia. Para lograr esa sinergia entre legalidad y legitimidad, se necesitan reformar los principales instrumentos legales en cuanto a la planificación, ordenamiento territorial, uso y gestión de suelo, para lograr una mayor cohesión en la elaboración de los planes.

Este artículo, bajo el planteamiento de la hipótesis: ¿Es suficientemente aplicable la normativa ecuatoriana para la ejecución de los Planes de Desarrollo y Ordenamiento Territorial Cantonales?, plantea un análisis y reflexión sobre la aplicabilidad de los preceptos jurídicos de la legislación nacional, dentro del ámbito y las competencias exclusivas de los Gobiernos Autónomos Descentralizados cantonales, para planificar el desarrollo y ordenamiento de su circunscripción territorial. La investigación obedece a los siguientes objetivos específicos ${ }^{2}$ :

- Estudiar teóricamente sobre la planificación en el desarrollo local;

- Identificar los cuerpos normativos aplicables y las competencias constitucionales y legales de los Gobiernos Autónomos Descentralizados Municipales, para la elaboración de políticas públicas a nivel cantonal;

- Caracterizar el proceso de actualización del Plan de Desarrollo y Ordenamiento Territorial del cantón Cuenca; y,

- Analizar la aplicación de la normativa jurídica ecuatoriana, en el proceso de actualización y ejecución del Plan de Desarrollo y Ordenamiento Territorial, según el caso de estudio del Gobierno Autónomo Descentralizado Municipal del cantón Cuenca.

En el primer capítulo, se tratará sobre los principales elementos teóricos de la planificación y el desarrollo territorial, dentro de las concepciones nacionales e internacionales, a fin de comprender los conceptos más relevantes que han sido fundamento para la planificación y la ordenación territorial ecuatoriana; en el segundo capítulo, se abordará sobre las disposiciones constitucionales y legales en cuanto a la planificación y el ordenamiento territorial, así como aquellas que versan sobre la competencia de los Gobiernos Autónomos Descentralizados cantonales; para que, finalmente, en un tercer capítulo, se analice si la normativa vigente, esta apegada a la realidad del territorio nacional y los Gobiernos Autónomos Descentralizados (GAD) cantonales. Para ello, se ha tomado como caso de estudio el GAD del cantón Cuenca.

\section{Metodología:}

La investigación del presente estudio, es de carácter cualitativa, desarrollada mediante un enfoque de teoría fundamentada. Esto permitirá realizar un contraste entre la normativa vigente y el proceso de actualización del Plan de Desarrollo y Ordenamiento Territorial

\footnotetext{
${ }^{2}$ Objetivos planteados por el autor, dentro de la estructura del marco teórico.
} 
dentro del cantón de estudio. Estableciendo un diagnóstico de operatividad y aplicabilidad de los preceptos legales del ordenamiento jurídico ecuatoriano; para posteriormente, según un alcance explicativo de la investigación, se pueda identificar las falencias técnico-jurídicas que limitan la construcción del Plan de Desarrollo y Ordenamiento Territorial cantonal.

Se utilizarán métodos teóricos, como el analítico-sintético, donde se consultarán bases de datos científicas y se revisará bibliografía relacionada con el objeto de estudio, a fin de obtener las bases teóricas y doctrinarias para la investigación.

Para establecer un diagnóstico situacional, se utilizará el método empírico como revisión documental y recolección de información. Asimismo, se realizarán entrevistas no estructuradas a expertos (funcionarios y ex funcionarios del Gobierno Autónomo Descentralizado de Cuenca), como un método de muestreo cualitativo, que permita una orientación correcta de la problemática actual. De similar forma, se analizará como muestreo de tipo, el caso específico del Gobierno Autónomo Descentralizado del cantón Cuenca y la actualización del Plan de Desarrollo y Ordenamiento Territorial 2020.

\section{La planificación y el desarrollo territorial: elementos teóricos. -}

Al abordar el concepto de desarrollo, por lo general suele asociarse a la descripción del crecimiento económico de un determinado territorio. Desde esta perspectiva, encontramos concensos importantes sobre teorías que han permitido ilustrar la organización territorial y el crecimiento económico.

Las teorías de localización, abordan sobre la relación entre los procesos económicos y el espacio territorial como una variable importante en el desarrollo económico. En esta línea, las teorías de los lugares centrales indican que, los servicios y bienes especializados son suministrados por las ciudades centrales urbanas.

A más de estas doctrinas clásicas, han existido conceptualizaciones que, nos han permitido evolucionar en cuanto al criterio de desarrollo; como indica López (2003), dentro de las consideraciones que hace la economía regional, se tiene como hipótesis de mayor afluencia a la teoría de la base de exportación, la teoría de las etapas de desarrollo, las teorías de los polos de desarrollo, las teorías de la causalidad circular y acumulativa.

Por otra parte, los llamados nuevos enfoques económicos, han dado origen a nuevos modelos económicos y de interrelación entre el capital y el territorio, donde destacan la nueva geografía económica, el postfordismo, el de competitividad y, el desarrollo desde abajo (López, J., 2003, p.55-83). 
Definir de manera absoluta el desarrollo local, sería entrar en ambigüedades vanas, dado que la construcción social y el desarrollo racional, han generado múltiples polaridades que de cierta forma abarcan gran parte de las características que deben tratarse en cuanto al desarrollo en sí mismo.

Para Barroso y Flores (2010), existen diversas variables que ameritan tratamiento especial, como recursos locales, participación social, participación empresarial, entre otros aspectos; empero, es necesario "identificar las condiciones generales que deberían estar presente en todo momento en un proceso de desarrollo local” (p. 62).

En tal sentido, coligen Barroso y Flores (2010) que, desarrollo local es "el conjunto de actuaciones concretas que definen los distintos agentes sociales, económicos, públicos o privados de la zona para reconducir los cambios estructurales que necesita un territorio concreto para conseguir solucionar su problemática mediante sus propios recursos" (p. $63)$.

Dentro de la planificación territorial, existen concepciones teóricas y doctrinarias que son útiles para estructurar la base de la presente investigación. Jeny López (2003), citando a Antonio Zárate, aborda las teorías de la estructura urbana, entendida ésta como "la especialización del suelo de la ciudad en zonas diferenciadas por sus características demográficas y sociales, y por las actividades predominantes en ellas" (p. 106) y, las divide en: a) teorías cásicas, como la teoría concéntrica, la teoría sectorial y, la teoría de los núcleos múltiples; y, b) teorías recientes, como las teorías del análisis de áreas sociales, las teorías de los valores del suelo y, las teorías de usos parciales del suelo (2003, p.106-113).

Por su parte Manuel Benabent (2016), indica que existe una escaza teorización en lo que respecta a la planificación, entendida esta como los procedimientos sobre la toma de decisiones. Otrora, la planificación versaba sobre un diseño trazado sobre el papel, donde no mediaba ningún criterio económico ni recursos locales, más bien, quedaba a discreción del dibujante, de su experticia y buen oficio; y, por supuesto la decisión y ejecución quedaba al arbitrio de otro versado en la rama.

El paso a una estructuración mejor planificada, dio lugar a una metodología de planificación racional, basada precisamente en el método científico, de allí que, el planificador, trabaja con la misma rigurosidad que la de un investigador científico, para desarollar el proceso planificador. Sin embargo, surgen críticas y posturas a esta planificación racional.

El llamado incrementalismo, surge como una antítesis al planteamiento puramente racionalista, considerando a la planificación como un conjunto de decisiones estratégicas sucesivas, orientadas a solventar los problemas que puedan surgir en los procesos de 
planificación, otorgándole con mayor solvencia el carácter de estratégica (2016, p. 354$358)$.

De igual forma, tras el desarrollo de estos planteamientos, surgen diversas perspectivas de teorización en cuanto a la planificación, donde es ineludible el involucramiento de la participación social para el desarrollo de nuevos lineamientos y campo de acción.

\section{La normativa ecuatoriana en la planificación y el ordenamiento territorial. -}

La Constitución de la República del Ecuador (2008), en su artículo 1, indica que, el Ecuador se gobierna de manera descentralizada. Prevé la organización territorial dentro del país; su sistema de integración se compone entre regiones, provincias, distritos metropolitanos, cantones y parroquias rurales. Y en este sentido, uno de los subsistemas de esta organización territorial, constituye el marco político, jurídico e institucional, como relaciones humanas que se proyecten a materializar los objetivos del ordenamiento territorial (Pauta, 2013, p.171).

Pese a este sistema territorial establecido en la Constitución, tenemos 24 provincias, 221 cantones y 881 parroquias rurales, pues en cuanto a las regiones y los distritos metropolitanos, no se ha logrado conformar de manera objetiva (Secretaría Nacional de Planificación y Desarrollo, 2012).

Precisamente, el artículo 238 de la Constitución de la República aborda lo concerniente a la autonomía política, administrativa y financiera que debe regir en los gobiernos autónomos descentralizados. En tal sentido, la Carta Magna en el artículo 264, establece las competencias exclusivas que, son atribuidas a los Gobiernos Autónomos Descentralizados-GAD cantonales, entre ellas, la de "planificar el desarrollo del cantón y formular los correspondientes planes de ordenamiento territorial" (Constitución de la República del Ecuador, 2008).

Dicho esto, para la operatividad y la planificación en estas escalas, como señala Pauta (2013), en el Ecuador "se han formulado el Plan Nacional de Desarrollo y los Planes de Desarrollo Regional, Provincial, Cantonal y Parroquial Rural, planes que establecerán... las directrices sociales, económicas, políticas, culturales y ambientales que orientarán el desarrollo humano en el espacio nacional y en los espacios regional, provincial, cantonal y parroquial rural" (p. 173).

En lo que respecta a la autonomía, como un principio rector para el ordenamiento territorial, señalado así en el artículo 5, numeral 3 de la LOOTUGS-Ley Orgánica de Ordenamiento Territorial, Uso y Gestión de Suelo (2016), donde expresamente expone que, los Gobiernos Autónomos Descentralizados ejercerán sus competencias sobre el ordenamiento territorial, en torno a las disposiciones constitucionales, legales y las regulaciones emitidas para el efecto. 
En cuanto al ordenamiento territorial, en armonía a lo dispuesto en el artículo 241 de la Constitución, la LOOTUGS (2016) en su artículo 9 indica que, "es el proceso y resultado de organizar espacial y funcionalmente las actividades y recursos en el territorio, para viabilizar la aplicación y concreción de políticas públicas democráticas y participativas y facilitar el logro de los objetivos de desarrollo" (Ley Orgánica de Ordenamiento Territorial, Uso y Gestión de Suelo, 2016). Este proceso, sin duda deberá constar en el plan de desarrollo de los Gobiernos Autónomos Descentralizados.

Sobre esto, Benabent y Vivanco (2018) precisan que el ordenamiento territorial, debe propender a cuatro objetivos centrales: " $a$ ) Resolver o prevenir conflictos de uso de las tierras... b) Propiciar el aprovechamiento sostenible de los recursos naturales... c) Reducir o evitar la ocurrencia de catástrofes... d) Resolver los desequilibrios del desarrollo económico regional y la fragmentación territorial...” (p. 718).

Para materializar lo establecido en la Constitución ecuatoriana, el Código Orgánico de Planificación y Finanzas Públicas (2010), en la sección tercera, define lo que debe entenderse como los planes de desarrollo y ordenamiento territorial, además de las directrices y contenidos que deben constar en los mismos. Asimismo, dentro del mentado cuerpo normativo, se estableció el término perentorio para que los Gobiernos Autónomos Descentralizados, presenten sus Planes de Desarrollo y Ordenamiento Territorial respectivo, bajo la condicionalidad de no aprobar las proformas presupuestarias quienes contrario sensu, no presenten dichos planes; pues, el artículo 49, establece la obligatoriedad de presentar dichos instrumentos, a fin de elaborar el presupuesto de cada GAD.

Por otro lado, el numeral 3 del artículo 11 de la Ley Orgánica de Ordenamiento Territorial, Uso y Gestión de Suelo (2016), indica que, los Gobiernos Autónomos Descentralizados cantonales, clasificarán el suelo del cantón en urbano y rural y, definirán su uso y gestión. Asimismo, sobre la base de esta decisión y estructura, podrán realizar sus intervenciones lo diferentes niveles de gobierno. Concomitante a ello es la disposición contenida en el literal b) del artículo 44 del Código Orgánico de Planificación y Finanzas Públicas (2010).

A pesar de las disposiciones normativas, la naciente etapa de planificación territorial, ha presentado complicaciones de orden práctico. González y Chuquiguanga, en el caso de la planificación de la zona 6 (Azuay, Cañar y Morona Santiago), revelan que apenas el 29\% de los cantones pertenecientes a la zona, cumplieron con la disposición transitoria cuarta del Código Orgánico de Planificación y Finanzas Públicas, esto es, haber presentado el Plan de Desarrollo y Ordenamiento Territorial hasta el 31 de diciembre de 2011. (2018) Luego de concluido el término, a pesar del incumplimiento, se otorgó el presupuesto a los GAD cantonales que no presentaron la aprobación de los planes. 


\section{La planificación y el ordenamiento territorial en el cantón Cuenca. -}

Al cantón lo dirige política y administrativamente, el Gobierno Autónomo Descentralizado Municipal de Cuenca. La representación de esta entidad, la ejerce el alcalde de la ciudad; quien, asimismo, preside el Concejo Municipal del cantón, donde además lo integran quince concejales, siendo esta entidad (Concejo Municipal) la que estructura y modifica las ordenanzas. Las competencias y atribuciones del Gobierno Autónomo Descentralizado de Cuenca surgen de la Constitución de la República y la Ley.

En cuanto al ámbito espacial, han surgido diversas teorías de orden territorial. Dentro del caso de estudio, el cantón Cuenca se constituye como una ciudad de tipo intelectual y religiosa (López, 2003), pues su estructura desde la época prehispánica, fue caracterizada por la edificación de templos y palacios; luego, tras la fundación española, durante la época colonial, se levantaron templos y monasterios de los cuales se conservan vestigios que, en algunos casos aún cumplen su función. En la misma línea, la ciudad de Cuenca ha sido declarada en el año 2011 como "Ciudad Universitaria" del Ecuador, emplazándose en la urbe cuatro instituciones de educación superior que acogen a estudiantes de diferentes partes del país.

En la Administración del año 2014, se actualiza por vez primera el Plan de Desarrollo y Ordenamiento Territorial del cantón, tras un diagnóstico basado en los componentes establecidos por la Secretaría Técnica Planifica Ecuador-SENPLADES ${ }^{3}$. Este plan, determina las potencialidades y los problemas dentro de la planificación territorial; jerarquizados a través de una matriz de Vester, donde se identifican problemas relacionados o derivados de la débil o nula aplicabilidad de la normativa jurídica ecuatoriana (Ilustre Municipalidad de Cuenca, 2016, p. 15).

Es necesario abordar desde una perspectiva jurídica, las principales limitantes que surgieron y se mantienen en la estructura del Plan de Desarrollo y Ordenamiento Territorial del caso de estudio de la presente investigación.

A fin de caracterizar la ejecución y el proceso de actualización del Plan de Desarrollo y Ordenamiento Territorial dentro del caso de estudio, se ha elaborado una entrevista no estructurada, donde se ha consultado a expertos en el área de planificación, Arquitecto Josué Vega, ex Director de Planificación del GAD cantonal de Cuenca en la actual administración; Arquitecto Pablo Sigüenza, Coordinador de la Unidad de Gestión Estratégica del GAD cantonal de Cuenca, área encargada de la actualización del Plan de Desarrollo y Ordenamiento Territorial cantonal; y, Arquitecto Adrián Parra, Coordinador del Equipo Técnico para elaborar el Plan de Uso y Gestión de Suelo del GAD cantonal de Cuenca. Entre los datos más relevantes que han proporcionado los entrevistados, se ha

\footnotetext{
${ }^{3}$ Los sistemas de análisis, se encuentran detalladamente abordados en el PDOT del cantón Cuenca.
} 
podido obtener la siguiente información (El autor, entrevista no estructurada, 26 de noviembre 2020):

Dentro del Gobierno Autónomo Descentralizado, ha existido una cultura de planificación, incluso antes de reformarse la Constitución de la República. A partir del año 2010 con la entrada en vigencia del Código Orgánico de Organización Territorial, Autonomía y Descentralización y el Código Orgánico de Planificación y Finanzas Públicas, se realiza el Plan de Desarrollo y Ordenamiento Territorial del cantón, el cual es aprobado en el año 2011 con proyección al año 2030 según el modelo deseado. En el año 2014 se realiza la primera actualización. Dentro de esta ejecución (2014), debido a las disposiciones del ente regulador de aquel entonces, la SENPLADES, la instrumentación se centraba más en el suelo rural. Esto también ocasionó una divergencia de criterios técnicos que llevaron consigo la asignación discrecional de permisos de construcción dentro de la urbe.

La normativa jurídica no es clara, se presta a la discrecionalidad técnica. La Ley Orgánica de Ordenamiento Territorial, Uso y Gestión de Suelo, esta dirigida fuertemente para su aplicación en el suelo urbano, con puntos muy básicos para la aplicación en el suelo rural. Para ejecutar planes y proyectos, ha sido necesario ampararse en cuerpos legales supletorios, lo cual también ha significado un sacrificio de la autonomía que deberían tener los Gobiernos Autónomos Descentralizados cantonales para la planificación, uso y regulación de suelo.

Los conceptos que establece sobre "uso de suelo" la Ley Orgánica de Ordenamiento Territorial, Uso y Gestión de Suelo, en el caso del GAD cantonal de Cuenca, se han tomado como conceptos generales; pero, para determinar un catalogo completo y nomenclatura específica, se han basado en la experiencia y experticia de los técnicos del $\mathrm{GAD}$, lo cual ha permitido una organización en cuanto a las construcciones y permisos.

Se presentan dificultades en la demarcación de zonas homogéneas y polígonos de intervención territorial, por cuanto la Ley Orgánica de Ordenamiento Territorial, Uso y Gestión de Suelo y su Reglamento, establecen preceptos muy generales, no toma en cuenta la realidad diferenciada del territorio nacional y la capacidad de análisis de información de los Gobiernos Autónomos Descentralizados.

Existen dificultades con respecto al trabajo cooperativo con otras instituciones del Gobierno Central para articular el proceso de actualización del Plan de Desarrollo y Ordenamiento Territorial, en el caso de áreas de protección, es necesario articular los procesos con el ente regulador nacional, el Ministerio del Ambiente; lo propio sucede de igual forma en cuanto a la planificación vial. Otra falencia es la falta de información en las entidades estatales, por ejemplo, se ha solicitado al Ministerio de Agricultura y Ganadería un catastro agrícola y productivo, sin embargo, no cuentan con el mismo, a la fecha, se encuentran apenas levantando información.

Previo a la vigencia de la Ley Orgánica de Ordenamiento Territorial, Uso y Gestión de Suelo, el marco legal era mucho más general, lo cual facilitaba la planificación, simplemente se requería que el instrumento de planificación contenga tres aspectos fundamentales, diagnóstico, propuesta y modelo de gestión; en torno a ello, la SENPLADES elaboró la guía metodológica a través del análisis por componentes, con el 
fin de que los procesos se unifiquen a nivel nacional. En el caso del Gobierno Autónomo Descentralizado cantonal de Cuenca, no se ha tomado en su totalidad lo contenido en la guía metodológica, han existido variables que se han incluido en instrumento de planificación cantonal, concomitantes con la guía y, otras que no se las ha incluido; sin embargo, la mayor parte del contenido del Plan de Desarrollo y Ordenamiento Territorial es acorde a la guía, con el fin de mantener cierta armonía con otros niveles de gobierno y Gobiernos Autónomos Descentralizados análogos.

Las instituciones públicas que participan en la planificación, como es el caso de la ex SENPLADES, hoy Planifica Ecuador y, el MIDUVI, han emitido disposiciones e instrumentos técnicos de manera tardía.

El Gobierno Autónomo Descentralizado cantonal de Cuenca, se encuentra trabajando en la unificación de sus planes de ordenamiento territorial, en lo que se denominará Plan de Desarrollo, Ordenamiento Territorial, Uso y Gestión de Suelo, es decir, tanto Plan de Desarrollo y Ordenamiento Territorial y, Plan de Uso y Gestión de Suelo formarán un solo instrumento.

\section{Resultados y discusión:}

El marco jurídico ecuatoriano, pretende ser lo suficientemente claro para garantizar la creación e instrumentación de políticas públicas que desarrollan los Gobiernos Autónomos Descentralizados cantonales en torno a su planificación territorial. Sin embargo, desde la vigencia de la Ley Orgánica de Ordenamiento Territorial, Uso y Gestión de Suelo, han surgido más dudas que certezas en lo que respecta al contenido de los planes de desarrollo y ordenamiento territorial cantonales.

Los planes de desarrollo y ordenamiento territorial, a los que refieren los artículos 41 y 43 del Código Orgánico de Planificación y Finanzas Públicas (2010), deberán contener mínimamente lo determinado en el artículo 42 ibídem; y, conforme determina la Ley Orgánica de Ordenamiento Territorial, Uso y Gestión de Suelo (2016) en el artículo 27, los planes también contendrán un Plan de Uso y Gestión de Suelo, el cual debe incluir los componentes estructurante y urbanístico, es decir, como lo señalan Benabent y Vivanco (2018), metodológicamente existiría un plan dentro de otro plan.

Además, este Plan de Uso y Gestión de Suelo, conforme lo determina el artículo 31 de la Ley Orgánica de Ordenamiento Territorial, Uso y Gestión de Suelo (2016), puede contener planes urbanísticos complementarios que, podrán brindar mayor especificidad a sus determinaciones. Estos planes complementarios, dice la norma, estarán subordinados al Plan de Desarrollo y Ordenamiento Territorial y, no podrán cambiar el contenido del Plan de Uso y Gestión de Suelo; lo cual deja una incertidumbre sobre el alcance normativo, pues, los planes de desarrollo y ordenamiento territorial cantonales, deben ser actualizados cada inicio de gestión del gobierno local, así lo dispone el artículo 48 del Código Orgánico de Planificación y Finanzas Públicas (2010) y, los planes de uso y gestión de suelo, a pesar de que pueden ser reformados, tienen una vigencia de doce años, 
según lo dispuesto en el artículo 30 de la Ley Orgánica de Ordenamiento Territorial, Uso y Gestión de Suelo (2016), esto ciertamente es una limitante para cada ejercicio de gestión.

En otra orilla, se encuentran las provincias de Morona Santiago, Napo, Orellana, Pastaza, Sucumbíos y Zamora Chinchipe que, por mandato constitucional y legal, su régimen de planificación y ordenamiento territorial, está regido por una Ley Especial ${ }^{4}$, desde luego, esto sin duda merecerá otro análisis e investigación.

Por otro lado, los Gobiernos Autónomos Descentralizados cantonales, enfrentan la dura tarea de coordinar con otras instituciones del gobierno central, para lograr consensos en cuanto a la planificación del territorio. En la praxis, se dificulta la estructuración de la planificación, debido a la poca claridad de los preceptos legales. La Constitución y la Ley, otorgan la competencia y la autonomía a los Gobiernos Autónomos Descentralizados cantonales, para la elaboración de los planes de ordenamiento territorial, así se desprende de los artículos 238, 264 numeral 1 de la Carta Magna (Constitución de la República del Ecuador, 2008) y, artículo 5 numeral 3 de la Ley Orgánica de Ordenamiento Territorial, Uso y Gestión de Suelo (2016). Sin embargo, indica la norma que, la planificación se la debe hacer con las diferentes instituciones del sector público involucradas y, con diversos actores sociales; de similar forma, prevé que la articulación de dichos planes, debe hacerse en concordancia a la planificación nacional, regional, provincial y parroquial. En otras palabras, todos los niveles de gobierno mantienen competencias ${ }^{5}$, lo cual dificulta la coordinación entre los diferentes actores involucrados.

Si bien es cierto, los Gobiernos Autónomos Descentralizados cantonales tienen la facultad de formular los planes de ordenamiento territorial y ejercer el control sobre el uso y ocupación de suelo dentro del cantón, sin embargo, dentro de su circunscripción existen otras entidades estatales que también ejercen control que, de cierta forma, limitan el ejercicio sobre la planificación; como se observó dentro del caso de estudio, sobre aquellas competencias de la Autoridad Agraria Nacional que, ejerce rectoría en el territorio rural, conforme lo dispone el artículo 4 de la Ley Orgánica de Tierras Rurales y Territorios Ancestrales (2016); o de aquellas zonas de protección o conservación, cuyo control lo ejerce el Estado central, a través de la Autoridad Ambiental Nacional, según lo dispone el numeral 7 del artículo 261 de la Constitución (2008); o las referentes a la vialidad, cuya competencia radica en diferentes niveles de gobierno, según se desprende de los 262, 263, 264, 267 y 314 de la Constitución (2008). Para dirimir sobre conflictos relacionados a la competencia, por vía administrativa, se ha creado el Consejo Nacional de Competencias, con fundamento en el artículo 269 de la Constitución (2008), cuyas

\footnotetext{
${ }^{4}$ Ley Orgánica para la Planificación Integral de la Circunscripción Territorial Especial Amazónica. https://www.sot.gob.ec/sotadmin2/_lib/file/doc/Ley_Orgánica\%20para\%20la\%20planificación\%20integr al $\% 20 \mathrm{de} \% 201 \mathrm{a} \% 20$ circunscripcion $\% 20$ territorial $\% 20$ especial $\% 20$ amazonica.Pdf

${ }^{5}$ Artículos 260 al 269 de la Constitución, sobre el régimen de competencias.
} 
funciones las señala el artículo 119 del Código Orgánico de Organización Territorial, Autonomía y Descentralización (2010).

A esto también se añade la falta de información o información desactualizada que, como de similar forma se pudo apreciar en el caso de estudio, no permiten a los técnicos trabajar en base a datos y catastros reales. De lo ratificado por los expertos, se desprende que, en la práctica y ejecución, ha sido necesario implementar instrumentos auxiliares y procesos, orientados a posibilitar la elaboración de los planes y que, estos a su vez, guarden armonía con el marco jurídico ecuatoriano.

Es oportuno colegir entonces, evidentemente existe una falta de especificidad jurídica que, permita o facilite una correcta articulación conjunta entre los actores de la planificación y el ordenamiento territorial que, deben ser tratados por el ordenamiento jurídico de la materia, sin menoscabo de las competencias de cada nivel de gobierno. El trabajo técnico en la elaboración de los planes de ordenamiento, debe sujetarse a lo tipificado en la norma, pero, surgen otras hipótesis que pueden y deben ser absueltas jurídicamente: ¿qué hacer cuando la realidad territorial difiere de la norma expresa o, a la inversa? ¿pueden los Gobiernos Autónomos Descentralizados cantonales crear instrumentos alternos que faciliten la elaboración de dichos planes?

\section{Conclusiones:}

- El proceso de planificación y ordenamiento territorial en el Ecuador es relativamente nuevo; los primeros planes a nivel general, se hicieron a partir del año 2011. Actualmente los Gobiernos Autónomos Descentralizados se encuentran en proceso de actualización por tercera ocasión. Sin los mecanismos e instrumentos adecuados de articulación conjunta, sumado a la falta de información actualizada, se generan conflictos de fondo que pueden afectar los procesos de ordenamiento territorial, uso y gestión de suelo.

- Se han creado constantemente diversos instrumentos técnicos y jurídicos por parte de los entes de control de manera posterior a la vigencia de la norma, a fin de viabilizar el trabajo en todos los niveles de gobierno, como son, por ejemplo, los orientados al uso y gestión de suelo, ello ocasiona cambios recurrentes en los procesos internos de los Gobiernos Autónomos Descentralizados.

- No existe un procedimiento legal determinado expresamente, para resolver los conflictos que versan sobre las competencias entre los niveles de gobierno. No se conoce con exactitud si deben llevarse de oficio por el ente regulador, en este caso, por el Consejo Nacional de Competencias o, si debe existir impulso de las partes.

- Si bien es cierto, la Constitución y la Ley otorgan competencias y autonomía a los Gobiernos Autónomos Descentralizados, para la planificación de su espacio; esta competencia se ve limitada por aquellas competencias atribuidas a los otros niveles de gobierno.

- Dentro del caso de estudio, pese a haber existido un antecedente de planificación en administraciones previas a la vigencia de la Constitución del 2008, del Código Orgánico de Organización Territorial, Autonomía y Descentralización, de la Ley Orgánica de Ordenamiento Territorial, Uso y Gestión de Suelo, entre otros cuerpos legales; aún 
mantienen conflictos en cuanto a la aplicación precisa de los instrumentos diseñados por el entre regulador.

\section{Referencias Bibliográficas}

Atienza, M., (2009). El sentido del Derecho (5ta ed.). Editorial Ariel. https://kupdf.net/queue/el-sentido-del-derecho-manuelatienza_58eff85adc0d60f73bda9805_pdf?queue_id=$1 \& x=1586974789 \& z=M T g 2 L j Q z L j E z N S 4 x N D g$

Barroso, M. y Flores, D. (2010). Teorías y estrategias de Desarrollo Local. Universidad Internacional de Andalucía. https://dspace.unia.es/bitstream/handle/10334/3817/2010_desarrollolocal.pdf

Benabent, M. (2016). Teorías de la planificación territorial: métodos de decisión. Ciudad y Territorio Estudios Territoriales. Volumen XLVIII, num. 189, 353-368. https://www.researchgate.net/publication/315685139_Teorias_de_la_planificaci on_territorial_metodos_de_decision

Benabent, M. y Vivanco L., (2018). El ordenamiento territorial y el urbanismo en el Ecuador $y$ su articulación competencial. https://www.researchgate.net/publication/326415582_El_ordenamiento_territori al_y_el_urbanismo_en_el_Ecuador_y_su_articulacion_competencial

Benabent, M. y Vivanco, L. (2019). La experiencia de los Planes de Desarrollo y Ordenamiento Territorial cantonales en Ecuador. Estoa, Revista de la Facultad de Arquitectura y Urbanismo de la Universidad de Cuenca, 8(15), 133-144. http://scielo.senescyt.gob.ec/pdf/estoa/v8n15/1390-9274-estoa-8-15-00229.pdf

Código Orgánico de Organización Territorial, Autonomía y Descentralización. (2010, 19 de octubre). Asamblea Nacional del Ecuador. Registro Oficial Sumplemento No. 303. http://www.lexis.com.ec/wp-content/uploads/2020/01/LI-Codigo-Organicode-Organizacion-Territorial-y-Descentralizacion-COOTAD.pdf

Código Orgánico de Planificación y Finanzas Públicas. (2010, 22 de octubre). Asamblea Nacional del Ecuador. Registro Oficial No. 306, Segundo Suplemento. https://amevirtual.gob.ec/wp-content/uploads/2017/04/09-CODIGOORGANICO-DE-PLANIFICACION-Y-FINANZAS-PUBLICAS-COPFP-1.pdf

Constitución de la República del Ecuador. (2008, 20 de octubre). Asamblea Nacional del Ecuador. Decreto Legislativo $0 . \quad$ Registro Oficial 449. https://www.oas.org/juridico/pdfs/mesicic4_ecu_const.pdf

González, M. y Chuquiguanga, M., (2018). Los Planes de Desarrollo y Ordenación Territorial en Ecuador a nivel cantonal, Estudio de caso de la Zona 6 de planificación. Estoa, Revista de la Facultad de Arquitectura y Urbanismo de la Universidad de Cuenca, 7(13), 101-110. https://publicaciones.ucuenca.edu.ec/ojs/index.php/estoa/article/view/1903/1393

Ilustre Municipalidad de Cuenca. (2016). Plan de Desarrollo y Ordenamiento Territorial 
de Cuenca. http://www.cuenca.gov.ec/?q=page_planordenamiento

Ley Orgánica de Ordenamiento Territorial, Uso y Gestión de Suelo. (2016, 05 de julio). Asamblea Nacional del Ecuador. Registro Oficial Suplemento No. 790. http://extwprlegs1.fao.org/docs/pdf/ecu166410.pdf

Ley Orgánica de Tierras Rurales y Territorios Ancestrales. (2016, 14 de marzo). Asamblea Nacional del Ecuador. Ley 0. Registro Oficial Suplemento No. 711. https://www.ambiente.gob.ec/wp-content/uploads/downloads/2018/09/LeyOrganica-de-Tierras-Rurales-y-Territorios-Ancestrales.pdf

López, J., (2003). Teorías y Enfoques del Desarrollo Territorial. Escuela Superior de Administración Pública. Programa de Administración Pública Territorial. http://www.esap.edu.co/portal/wp-content/uploads/2017/10/5-Teorias-Enfoquesdel-Desarrollo-Territorial.pdf

Nino, C., (2003). Introducción al análisis del derecho (2da ed., 12a reimp.). Editorial Astrea.

https://inecipcba.files.wordpress.com/2013/08/introduccion_al_analisis_del_der echo_-_carlos_santiago_nino.pdf

Pauta, F., (2013). Ordenación territorial y urbanística:isepiun camino para su aplicación en el ecuador. Universidad de Cuenca. https://www.google.com/url?sa=t\&rct=j\&q=\&esrc=s\&source=web\&cd=1\&ved= 2ahUKEwi7-

Yn60O3oAhWFY98KHXmfAEQQFjAAegQIARAB\&url=https\%3A\%2F\%2Fd ialnet.unirioja.es\%2Fdescarga\%2Flibro\%2F693647.pdf\&usg=AOvVaw19EVkD cErA_zvGB3KCpiPY

Pauta, F., (2019). ¿Son técnicamente viables los planes de uso y gestión de suelo previstos por la ley ecuatoriana de ordenamiento territorial?. Eídos, [S.1.], v. 13, n. 1, p. 39 - 54. https://revistas.ute.edu.ec/index.php/eidos/article/view/543

Secretaría Nacional de Planificación y Desarrollo. (2012). Proceso de desconcentración del Ejecutivo en los niveles administrativos de planificación 1ra Edición. https://www.planificacion.gob.ec/wpcontent/uploads/downloads/2012/10/Folleto_informativoDesconcentracion2012.pdf

\section{\Cigital}




\section{PARA CITAR EL ARTÍCULO INDEXADO.}

Carpio Arévalo, J. A. (2021). La aplicabilidad de la normativa jurídica ecuatoriana, en torno a los PDOT cantonales. Estudio de caso del GAD Municipal del cantón Cuenca. ConcienciaDigital, 4(1.1), 6-21. https://doi.org/10.33262/concienciadigital.v4i1.1.1544

\section{\Ciencia}

El artículo que se publica es de exclusiva responsabilidad de los autores y no necesariamente reflejan el pensamiento de la Revista Conciencia Digital.

El artículo queda en propiedad de la revista y, por tanto, su publicación parcial y/o total en otro medio tiene que ser autorizado por el director de la Revista Conciencia Digital.

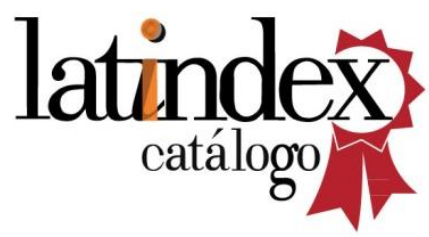

